\title{
Basic Properties of the Polygonal Sequence
}

\author{
Kunle Adegoke \\ adegoke00@gmail.com \\ Department of Physics and Engineering Physics, \\ Obafemi Awolowo University, 220005 Ile-Ife, Nigeria
}

2010 Mathematics Subject Classification: Primary 11B99; Secondary 11H99.

Keywords: polygonal number, triangular number, summation identity, generating function, partial sum, recurrence relation.

\begin{abstract}
We study various properties of the polygonal numbers; such as their recurrence relations, fundamental identities, weighted binomial and ordinary sums and the partial sums and generating functions of their powers. A feature of our results is that they are presented naturally in terms of the polygonal numbers themselves and not in terms of arbitrary integers as is the case in most literature.
\end{abstract}

\section{Introduction}

For a fixed integer $r \geq 2$, the polygonal sequence $\left(P_{n, r}\right)$ is defined through the recurrence relation,

$$
P_{n, r}=2 P_{n-1, r}-P_{n-2, r}+r-2(n \geq 2),
$$

with $P_{0, r}=0, P_{1, r}=1$.

Thus, the polygonal sequence is a second order, linear non-homogeneous sequence with constant coefficients. In $\S 1.2$ we shall see that the polygonal numbers can also be defined by a third order homogeneous recurrence relation.

The fixed number $r$ is called the order or rank of the polygonal sequence. The $n t h$ polygonal number of order $r, P_{n, r}$, derives its name from representing a polygon by dots in a plane: $r$ is the number of sides and $n$ is the number of dots on each side. Thus, for a triangular number, $r=3$, for a square number, $r=4$ (four sides), and so on.

The book by Deza and Deza [4] is an excellent source book on polygonal numbers and figurate numbers in general. Cook and Bacon [3] derived some summation formulas involving the polygonal numbers. Hoggatt and Bicknell [8] studied the triangular numbers. The article by Garge and Shirali [6] showcases some interesting basic properties of the triangular numbers. We refer the reader to Dickson [5, Chapter I] for an historical perspective on polygonal numbers. 
In the sequel, the equations look nicer and simpler if we characterize polygonal numbers by a quantity $\alpha(r)$ defined by $\alpha(r) \equiv r-2$. For brevity we will always write $\alpha$ when we mean $\alpha(r)$. The recurrence relation for polygonal numbers is then,

$$
P_{n, r}=2 P_{n-1, r}-P_{n-2, r}+\alpha(n \geq 2),
$$

with $P_{0, r}=0, P_{1, r}=1$.

We have $\alpha=0$ for natural numbers $\left(P_{n, 2}=n\right), \alpha=1$ for triangular numbers, $\alpha=2$ for square numbers and so on.

The difference equation (1.1) is readily solved, yielding an exact formula for the $n t h r$-gonal number, namely,

$$
P_{n, r}=\frac{\alpha}{2} n^{2}-\frac{(\alpha-2)}{2} n .
$$

The $n t h$ triangular number, $P_{n, 3}$, will, henceforth, be denoted by $T_{n}$ :

$$
T_{n}=P_{n, 3}=\frac{n(n+1)}{2} .
$$

By re-arranging the rhs of (1.2), we see that every polygonal number can be expressed in terms of a triangular number:

$$
P_{n, r}=n+\alpha T_{n-1}
$$

Since $T_{n}-T_{n-1}$, we also have

$$
P_{n, r}=T_{n}+(\alpha-1) T_{n-1}
$$

\subsection{Extension to negative subscripts}

As is the case with other integer sequences, it is possible to continue the polygonal sequence to include negative indices. Write the recurrence relation as

$$
P_{n, r}=2 P_{n+1, r}-P_{n+2, r}+\alpha
$$

and replace $n$ with $-n$, obtaining,

$$
P_{-n, r}=2 P_{-(n-1), r}-P_{-(n-2), r}+\alpha,
$$

for $n=1,2, \ldots$

This gives $P_{-1, r}=\alpha-1, P_{-2, r}=3 \alpha-2, P_{-3, r}=6 \alpha-3$, and so on.

The solution of the difference equation

$$
P_{m, r}=2 P_{m+1, r}-P_{m+2, r}+\alpha
$$

with initial conditions $P_{-1, r}=\alpha-1, P_{-2, r}=3 \alpha-2$, is

$$
P_{m, r}=\frac{\alpha}{2} m^{2}-\frac{(\alpha-2)}{2} m .
$$

Writing $-n$ for $m$ we have

$$
P_{-n, r}=\frac{\alpha}{2} n^{2}+\frac{(\alpha-2)}{2} n .
$$


Thus, the sequence $\left(P_{n, r}\right)$ is defined for all integers; with the exact values given by (1.2) and (1.6).

Note, in particular, that,

$$
T_{-n}=P_{-n, 3}=\frac{n^{2}}{2}+\frac{-n}{2}=\frac{(n-1) n}{2}=T_{n-1} .
$$

Addition of (1.2) and (1.6) gives

$$
P_{-n, r}=\alpha n^{2}-P_{n, r},
$$

while their difference gives

$$
P_{-n, r}=(\alpha-2) n+P_{n, r} .
$$

Writing $-n$ for $n$ in (1.3) and making use of (1.7), we also have

$$
P_{-n, r}=-n+\alpha T_{n} .
$$

\subsection{A third order linear recurrence relation}

Write the polygonal recurrence relation (1.1) in two ways,

$$
\begin{gathered}
P_{j, r}-2 P_{j-1, r}=\alpha-P_{j-2, r}, \\
2 P_{j, r}-P_{j-1, r}=P_{j+1, r}-\alpha,
\end{gathered}
$$

and add to obtain

$$
3 P_{j, r}-3 P_{j-1, r}=P_{j+1, r}-P_{j-2, r},
$$

which can be written

$$
P_{j, r}=3 P_{j-1, r}-3 P_{j-2, r}+P_{j-3, r} .
$$

Relation (1.13) is a special case of a more general recurrence relation, given in Corollary 6. Note that (1.13) with the seeds $P_{0, r}=0, P_{1, r}=1$ and $P_{2, r}=\alpha+2$ makes $\left(P_{n, r}\right)$ a third order recurrence sequence.

\subsection{Sum of the first $k$ polygonal numbers}

Theorem 1. If $k$ is an integer, then,

$$
\begin{gathered}
\sum_{j=0}^{k} P_{j, r}=\left(\frac{\alpha}{3}(k-1)+1\right) T_{k}, \\
\sum_{j=0}^{k}(-1)^{j} P_{j, r}=\left\{\begin{array}{l}
\frac{\alpha}{2} T_{k}-\frac{\alpha-2}{4} k, \quad \text { if } k \text { is even } ; \\
-\frac{\alpha}{2} T_{k}+\frac{\alpha-2}{4}(k+1), \quad \text { if } k \text { is odd } .
\end{array}\right.
\end{gathered}
$$


Proof. Using (1.2), we have

$$
\begin{gathered}
\sum_{j=0}^{k} P_{j, r}=\frac{\alpha}{2} \sum_{j=0}^{k} j^{2}-\frac{\alpha-2}{2} \sum_{j=0}^{k} j, \\
\sum_{j=0}^{k}(-1)^{j} P_{j, r}=\frac{\alpha}{2} \sum_{j=0}^{k}(-1)^{j} j^{2}-\frac{\alpha-2}{2} \sum_{j=0}^{k}(-1)^{j} j,
\end{gathered}
$$

from which identities (1.14) and (1.15) follow upon using the following well-known results:

$$
\begin{gathered}
\sum_{j=0}^{k} j=T_{k}, \quad \sum_{j=0}^{k} j^{2}=\frac{k(k+1)(2 k+1)}{6}=\frac{(2 k+1)}{3} T_{k}, \\
\sum_{j=0}^{k}(-1)^{j} j=\left\{\begin{array}{ll}
k / 2, & \text { if } k \text { is even, } \\
-(k+1) / 2, & \text { if } k \text { is odd, }
\end{array}, \quad \sum_{j=0}^{k}(-1)^{j} j^{2}= \begin{cases}T_{k}, & \text { if } k \text { is even, } \\
-T_{k}, & \text { if } k \text { is odd. }\end{cases} \right.
\end{gathered}
$$

We remark that, since,

$$
\sum_{j=0}^{k} e^{x j}=\frac{1-e^{(k+1) x}}{1-e^{x}}, \quad \sum_{j=0}^{k}(-1)^{j} e^{x j}=\frac{1+(-1)^{k} e^{(k+1) x}}{1+e^{x}}
$$

the sums of powers of consecutive integers are readily found from

$$
\sum_{j=0}^{k} j^{i}=\left.\frac{d^{i}}{d x^{i}}\left(\frac{1-e^{(k+1) x}}{1-e^{x}}\right)\right|_{x=0}, \quad \sum_{j=0}^{k}(-1)^{j} j^{i}=\left.\frac{d^{i}}{d x^{i}}\left(\frac{1+(-1)^{k} e^{(k+1) x}}{1+e^{x}}\right)\right|_{x=0} .
$$

For the triangular numbers, identities (1.14) and (1.15) reduce to

$$
\begin{gathered}
\sum_{j=0}^{k} T_{j}=\frac{k+2}{3} T_{k}=\frac{k(k+1)(k+2)}{6}, \\
\sum_{j=0}^{k}(-1)^{j} T_{j}=\left\{\begin{array}{l}
\frac{1}{2} T_{k}+\frac{k}{4}, \quad \text { if } k \text { is even } ; \\
-\frac{1}{2} T_{k}-\frac{k+1}{4}, \quad \text { if } k \text { is odd } .
\end{array}\right.
\end{gathered}
$$

\subsection{Some fundamental identities}

What Garge and Shirali [6] noted about triangular numbers is true about polygonal numbers in general: it is generally relatively easy to prove relations and identities involving polygonal numbers, often by simple algebra; the real challenge is in discovering them.

By using the exact formula (1.2), it is straightforward to verify the following identities involving the polygonal numbers:

$$
P_{n, r}-P_{n-1, r}=(n-1) \alpha+1
$$




$$
\begin{gathered}
P_{n, r}+P_{n-1, r}=(n-1)^{2} \alpha+2 n-1, \\
P_{j m, r}=P_{j, r} P_{m, r}-\alpha(\alpha-2) T_{m-1} T_{j-1}, \\
P_{m+n, r}-P_{m-n, r}=n\left(P_{m+1, r}-P_{m-1, r}\right), \\
P_{n+m, r}+P_{n-m, r}=2 P_{n, r}+\alpha m^{2}, \\
P_{m+n, r} P_{m-n, r}=\left(P_{m, r}-P_{n, r}\right)\left(P_{m, r}-P_{-n, r}\right), \\
P_{n+m, r}=(m+1) P_{n, r}-m P_{n-1, r}+\alpha T_{m}, \\
P_{n+j m, r}=P_{n, r}+j P_{m, r}+\alpha m^{2} T_{j-1}+\alpha m n j, \\
P_{n-j m, r}=P_{n, r}-j P_{m, r}+\alpha m^{2} T_{j}-\alpha m n j .
\end{gathered}
$$

Setting $m=j$ in (1.21), we have

$$
P_{j^{2}, r}=P_{j, r}^{2}-\alpha(\alpha-2) T_{j-1}^{2},
$$

which generalizes the well known triangular number identity,

$$
T_{j^{2}}=T_{j}^{2}+T_{j-1}^{2}
$$

For the triangular numbers $(\alpha=1)$, identities $(1.19)$ - (1.27) reduce to:

$$
\begin{gathered}
T_{n}-T_{n-1}=n, \\
T_{n}+T_{n-1}=n^{2}, \\
T_{j m}=T_{j} T_{m}+T_{m-1} T_{j-1}, \\
T_{m+n}-T_{m-n}=n\left(T_{m+1}-T_{m-1}\right), \\
T_{n+m}+T_{n-m}=2 T_{n}+m^{2}, \\
T_{n+m} T_{n-m}=\left(T_{n}-T_{m}\right)\left(T_{n}-T_{m-1}\right), \\
T_{n+m}=(m+1) T_{n}-m T_{n-1}+T_{m}, \\
T_{m j+n}=T_{n}+j T_{m}+m^{2} T_{j-1}+m n j, \\
T_{n-m j}=T_{n}-j T_{m}+m^{2} T_{j}-m n j .
\end{gathered}
$$

Note that the well known identity:

$$
T_{m+n}=T_{n}+T_{m}+m n,
$$

is a special case of (1.37). 


\subsection{Basic summation tools}

In the sequel, we will often make use of the index shift identity,

$$
\sum_{j=0}^{k} f_{j+a} x^{j}=x^{-a} \sum_{j=0}^{k} f_{j} x^{j}-x^{-a} \sum_{j=0}^{a-1} f_{j} x^{j}+x^{-a} \sum_{j=k+1}^{k+a} f_{j} x^{j}
$$

where $\left(f_{i}\right)$ is any real sequence and $a$ and $k$ are any integers.

Note that if $a<1$, then,

$$
\sum_{j=0}^{a-1} f_{j} x^{j} \equiv-\sum_{j=a}^{-1} f_{j} x^{j}
$$

and

$$
\sum_{j=k+1}^{k+a} f_{j} x^{j} \equiv-\sum_{j=k+a+1}^{k} f_{j} x^{j}
$$

We also require the following telescoping summation identities:

$$
\begin{gathered}
\sum_{j=0}^{k}\left(f_{j+1}-f_{j}\right)=f_{k+1}-f_{0}, \\
\sum_{j=0}^{k}(-1)^{j}\left(f_{j+1}+f_{j}\right)=(-1)^{k} f_{k+1}+f_{0},
\end{gathered}
$$

or, equivalently,

$$
\begin{gathered}
\sum_{j=1}^{k}\left(f_{j+1}-f_{j}\right)=f_{k+1}-f_{1}, \\
\sum_{j=1}^{k}(-1)^{j-1}\left(f_{j+1}+f_{j}\right)=(-1)^{k-1} f_{k+1}+f_{1} .
\end{gathered}
$$

Identity (1.45) is a special case of a more general telescoping summation formula, namely,

$$
\sum_{j=1}^{k}\left(f_{j+n}-f_{j}\right)=\sum_{j=1}^{n}\left(f_{j+k}-f_{j}\right)
$$

As an immediate application of (1.43), we can extract a summation identity from the recurrence relation $(1.12)$.

Theorem 2. If $k$ is an integer, then,

$$
\sum_{j=0}^{k} 2^{j} P_{j, r}=2^{k+1}\left(P_{k-1, r}+\alpha\right)+2(1-2 \alpha) .
$$

Proof. Write identity (1.12) as

$$
P_{j, r}-\alpha=2 P_{j-1, r}-P_{j-2, r}
$$


and multiply through by $2^{j-1}$ to obtain

$$
2^{j-1} P_{j, r}-\alpha 2^{j-1}=2^{j} P_{j-1, r}-2^{j-1} P_{j-2, r} .
$$

Thus,

$$
\sum_{j=0}^{k} 2^{j-1} P_{j, r}-\alpha \sum_{j=0}^{k} 2^{j-1}=\sum_{j=0}^{k}\left(2^{j} P_{j-1, r}-2^{j-1} P_{j-2, r}\right) .
$$

To evaluate the sum on the right hand side, identify $f_{j}=2^{j-1} P_{j-2, r}$ and use identity (1.43) to obtain

$$
\sum_{j=0}^{k}\left(2^{j} P_{j-1, r}-2^{j-1} P_{j-2, r}\right)=2^{k} P_{k-1, r}-\frac{1}{2}(3 \alpha-2) .
$$

Identity (1.48) now reads

$$
\sum_{j=0}^{k} 2^{j-1} P_{j, r}-\alpha \sum_{j=0}^{k} 2^{j-1}=2^{k} P_{k-1, r}-\frac{1}{2}(3 \alpha-2)
$$

or

$$
\sum_{j=0}^{k} 2^{j} P_{j, r}-\alpha \sum_{j=0}^{k} 2^{j}=2^{k+1} P_{k-1, r}-3 \alpha+2,
$$

from which the result follows when we insert the geometric sum $\sum_{j=0}^{k} 2^{j}=2^{k+1}-1$.

\section{A master identity}

Theorem 3. Let $a, b, c, d$ and $e$ be integers. Then,

$$
\begin{aligned}
& \left(T_{a-c} T_{c-b} T_{b-a}+T_{b-c} T_{c-a} T_{a-b}\right) P_{d+e, r} \\
& \begin{aligned}
=\quad & \left.T_{b-c} T_{c-a} T_{e-b}-T_{b-c} T_{c-b} T_{e-a}+T_{c-b} T_{b-a} T_{e-c}\right) P_{d+a, r} \\
& +\left(T_{a-c} T_{c-b} T_{e-a}-T_{a-c} T_{c-a} T_{e-b}+T_{c-a} T_{a-b} T_{e-c}\right) P_{d+b, r} \\
& \quad+\left(T_{b-c} T_{a-b} T_{e-a}-T_{a-b} T_{b-a} T_{e-c}+T_{a-c} T_{b-a} T_{e-b}\right) P_{d+c, r}
\end{aligned}
\end{aligned}
$$

Proof. We seek to express a polygonal number as a linear combination of three triangular numbers. Let

$$
P_{d+e, r}=f_{1} T_{e-a}+f_{2} T_{e-b}+f_{3} T_{e-c},
$$

where $a, b, c, d$ and $e$ are arbitrary integers and the coefficients $f_{1}, f_{2}$ and $f_{3}$ are to be determined. Setting $e=a, e=b$ and $e=c$, in turn, we obtain three simultaneous equations:

$$
P_{d+a, r}=f_{2} T_{a-b}+f_{3} T_{a-c}, \quad P_{d+b, r}=f_{1} T_{b-a}+f_{3} T_{b-c}, \quad P_{d+c, r}=f_{1} T_{c-a}+f_{2} T_{c-b} .
$$

The identity of Theorem 3 is established by solving these equations for $f_{1}, f_{2}$ and $f_{3}$ and substituting the solutions into identity (2.1).

Note that the identity of Theorem 3 can also be written

$$
\begin{aligned}
(a-b)(a-c)(b-c) P_{d+e, r}= & (b-c)(e-c)(e-b) P_{d+a, r} \\
& -(a-c)(e-a)(e-c) P_{d+b, r} \\
& +(a-b)(e-a)(e-b) P_{d+c, r} .
\end{aligned}
$$

Theorem 3 yields a myriad of recurrence relations, of which we can mention a couple. 
Corollary 4. Let $m$ and $j$ be integers. Then

$$
P_{j+m, r}=T_{m-1} P_{j+2, r}+\left(T_{m}-3 T_{m-1}\right) P_{j+1, r}+T_{m-2} P_{j, r} .
$$

Proof. Set $c=0, b=1, a=2, d=j, e=m$ in Theorem 3 .

In particular, we have the addition formula for the triangular numbers:

$$
T_{j+m}=T_{m-1} T_{j+2}+\left(T_{m}-3 T_{m-1}\right) T_{j+1}+T_{m-2} T_{j}
$$

Corollary 5. Let $j$, a and $e$ be integers. Then,

$$
\begin{aligned}
(j+a)(a-c)(j+c) P_{j+e, r}=(j+c)(e-c)(j+e) P_{j+a, r} \\
-(j+a)(e-a)(j+e) P_{j+c, r} .
\end{aligned}
$$

Proof. Set $d=-b$ followed by $b=-j$ in identity 2.2 .

Setting $n=0$ in Corollary 5 gives

$$
\left(c T_{a}-a T_{c}\right) P_{e, r}=\left(c T_{e}-e T_{c}\right) P_{a, r}+\left(e T_{a}-a T_{e}\right) P_{c, r} .
$$

Corollary 6. Let $m$ and $j$ be integers. Then,

$$
T_{m} P_{j+1, r}=T_{m+1} P_{j, r}-T_{m+1} P_{j-m, r}+T_{m} P_{j-m-1, r} .
$$

Proof. Set $a=0, e=1, b=-m, c=-m-1$ and $d=j$ in (2.2).

By writing $-m$ for $m$, the above identity can also be written

$$
T_{m-1} P_{j+1, r}=T_{m-2} P_{j, r}-T_{m-2} P_{j+m, r}+T_{m-1} P_{j+m-1} .
$$

In particular, we have

$$
T_{m} T_{j+1}=T_{m+1} T_{j}-T_{m+1} T_{j-m}+T_{m} T_{j-m-1}
$$

\section{Linear properties: partial sums and generating func- tions}

In this section, we will derive expressions for the partial sum of the polygonal numbers, namely, $\sum_{j=0}^{k} P_{j, r} x^{j}$ and hence obtain the generating function, $\sum_{j=0}^{\infty} P_{j, r} x^{j}$. Two equivalent expressions will be derived, one based on identity (1.12), the non homogeneous second order recurrence relation and the other based on identity (1.13), the homogeneous third order recurrence relation for polygonal numbers. We will also derive an expression for the partial sum of the polygonal numbers with indices in arithmetic progression, $\sum_{j=0}^{k} P_{m j+n, r} x^{j}$. 


\subsection{Partial sum of polygonal numbers}

Theorem 7. If $k$ is an integer, then,

$$
\sum_{j=0}^{k} P_{j, r} x^{j}=\frac{x}{(1-x)^{2}}\left(x^{k}\left(x P_{k, r}-P_{k+1, r}\right)+1-\alpha\right)+\frac{\alpha x}{(1-x)^{3}}\left(1-x^{k+1}\right) .
$$

Proof. Multiply the recurrence relation (1.12) by $x^{j}$ and sum over $j$ to obtain

$$
\sum_{j=0}^{k} \mathrm{P}_{\mathrm{j}+1, \mathrm{r}} x^{j}+\sum_{j=0}^{k} \mathrm{P}_{\mathrm{j}-1, \mathrm{r}} x^{j}-2 \sum_{j=0}^{k} \mathrm{P}_{\mathrm{j}, \mathrm{r}} x^{j}=\alpha \sum_{j=0}^{k} x^{j}
$$

in which,

$$
\begin{gathered}
\sum_{j=0}^{k} \mathrm{P}_{\mathrm{j}+1, \mathrm{r}} x^{j}=x^{k} P_{k+1, r}+\frac{1}{x} \sum_{j=0}^{k} \mathrm{P}_{\mathrm{j}, \mathrm{r}} x^{j}, \\
\sum_{j=0}^{k} \mathrm{P}_{\mathrm{j}-1, \mathrm{r}} x^{j}=\alpha-1-x^{k+1} P_{k, r}+x \sum_{j=0}^{k} \mathrm{P}_{\mathrm{j}, \mathrm{r}} x^{j},
\end{gathered}
$$

and

$$
\sum_{j=0}^{k} x^{j}=\frac{1-x^{k+1}}{1-x}
$$

Use of (3.2), (3.3) and (3.4) in (3.1) gives the stated identity.

For the triangular numbers, we have

$$
\sum_{j=0}^{k} T_{j} x^{j}=\frac{x^{k+1}}{(1-x)^{2}}\left(x T_{k}-T_{k+1}\right)+\frac{x}{(1-x)^{3}}\left(1-x^{k+1}\right) .
$$

Corollary 8. If $k$ is an integer, then,

$$
\begin{gathered}
\sum_{j=0}^{k} P_{j, r}=T_{k+1} P_{k, r}-T_{k} P_{k+1, r}+\frac{\alpha k T_{k+1}}{3}=-(\alpha-1) T_{k}+\frac{\alpha k T_{k+1}}{3}, \\
\sum_{j=0}^{k}(-1)^{j} P_{j, r}=\left\{\begin{array}{l}
\left(P_{k, r}+P_{k+1, r}-1\right) / 4, \quad \text { if } k \text { is even } \\
\left\{\alpha-\left(1+P_{k, r}+P_{k+1, r}\right)\right\} / 4, \quad \text { if } k \text { is odd } .
\end{array}\right.
\end{gathered}
$$

In particular,

$$
\begin{gathered}
\sum_{j=0}^{k} T_{j}=\frac{k(k+1)(k+2)}{6}=\frac{1}{3} k T_{k+1}, \\
\sum_{j=0}^{k}(-)^{j} T_{j}= \begin{cases}k(k+2) / 4, & \text { If } k \text { is even; } \\
-(k+1)^{2} / 4, & \text { If } k \text { is odd } .\end{cases}
\end{gathered}
$$

Corollary 9. We have

$$
\sum_{j=0}^{\infty} P_{j, r} x^{j}=\frac{(1-\alpha) x}{(1-x)^{2}}+\frac{\alpha x}{(1-x)^{3}}
$$


In particular,

$$
\sum_{j=0}^{\infty} T_{j} x^{j}=\frac{x}{(1-x)^{3}}
$$

Lemma 1 ([2, Lemma 2.3]Partial sum of a $n+1$-term sequence). Let $\left(X_{j}\right)$ be any arbitrary sequence, where $X_{j}, j \in \mathbb{Z}$, satisfies a $n+1$ term recurrence relation $X_{j}=f_{1} X_{j-c_{1}}+$ $f_{2} X_{j-c_{2}}+\cdots+f_{n} X_{j-c_{n}}=\sum_{m=1}^{n} f_{m} X_{j-c_{m}}$, where $f_{1}, f_{2}, \ldots, f_{n}$ are arbitrary non-vanishing complex functions, not dependent on $j$, and $c_{1}, c_{2}, \ldots, c_{n}$ are fixed integers. Then, the following summation identity holds for arbitrary $x$ and non-negative integer $k$ :

$$
\sum_{j=0}^{k} X_{j} x^{j}=\frac{\sum_{m=1}^{n}\left\{x^{c_{m}} f_{m}\left(\sum_{j=1}^{c_{m}} x^{-j} X_{-j}-\sum_{j=k-c_{m}+1}^{k} x^{j} X_{j}\right)\right\}}{1-\sum_{m=1}^{n} x^{c_{m}} f_{m}}
$$

Theorem 10. If $k$ is an integer, then,

$$
\begin{gathered}
\sum_{j=0}^{k} P_{j, r} x^{j}=\frac{x^{2}(\alpha-1)+x}{(1-x)^{3}}-\frac{\left\{(1-x)^{3}+x^{3}\right\}}{(1-x)^{3}} P_{k+1, r} x^{k+1} \\
-\frac{(1-3 x)}{(1-x)^{3}} P_{k+2, r} x^{k+2}-\frac{P_{k+3, r} x^{k+3}}{(1-x)^{3}}
\end{gathered}
$$

Proof. Arrange recurrence relation (1.13) as

$$
P_{j, r}=P_{j+3, r}-3 P_{j+2, r}+3 P_{j+1, r} .
$$

In Lemma 1 choose $\left(f_{1}, f_{2}, f_{3}\right)=(1,-3,3),\left(c_{1}, c_{2}, c_{3}\right)=(-3,-2,-1)$ and $\left(X_{j}\right)=\left(P_{j, r}\right)$.

Theorem 11. Let $m$ and $k$ be integers. Then

$$
\begin{aligned}
\sum_{j=0}^{k} P_{j+m, r} x^{j}=- & \frac{m(1-x)+2 x}{(m(1-x)-2)(1-x)^{2}}\left(x^{k}\left(x P_{k, r}-P_{k+1, r}\right)+1-\alpha\right) \\
& -\frac{\alpha(m(1-x)+2 x)}{(m(1-x)-2)(1-x)^{3}}\left(1-x^{k+1}\right) \\
& +\frac{m}{m(1-x)-2}\left(P_{m-1, r}-x^{k}\left(P_{k+1, r}+x P_{k+m, r}\right)\right) .
\end{aligned}
$$

Proof. Arrange identity (2.5) as

$$
T_{m-2} P_{j+m, r}-T_{m-1} P_{j+m-1, r}=T_{m-2} P_{j, r}-T_{m-1} P_{j+1, r}
$$

multiply through by $x^{j}$ and sum over $j$, obtaining,

$$
\begin{gathered}
x\left(T_{m-2}-x T_{m-1}\right) \sum_{j=0}^{k} P_{j+m, r} x^{j}=\left(x T_{m-2}-T_{m-1}\right) \sum_{j=0}^{k} P_{j, r} x^{j}-T_{m-1} x^{k+1} P_{k+1, r} \\
+x T_{m-1} P_{m-1, r}-x^{k+2} T_{m-1} P_{k+m, r} .
\end{gathered}
$$


Corollary 12. Let $k$ and $m$ be integers. Then,

$$
\begin{aligned}
& \sum_{j=0}^{k} P_{j+m, r}=-(\alpha-1) T_{k}+\frac{\alpha k T_{k+1}}{3}+\frac{m}{2}\left(P_{k+m, r}+P_{k+1, r}-P_{m-1, r}\right), \\
& \sum_{j=0}^{k}(-1)^{j} P_{j+m, r}=\left\{\begin{array}{l}
\frac{\left(P_{k, r}+P_{k+1, r}-1\right)}{4}+\frac{m}{2(m-1)}\left(P_{k+m, r}-P_{k+1, r}+P_{m-1, r}\right), \quad \text { if } k \text { is even } ; \\
\frac{\left\{\alpha-\left(1+P_{k, r}+P_{k+1, r}\right)\right\}}{4}-\frac{m}{2(m-1)}\left(P_{k+m, r}-P_{k+1, r}-P_{m-1, r}\right), \quad \text { if } k \text { is odd } .
\end{array}\right.
\end{aligned}
$$

\subsection{Partial sum of polygonal numbers with indices in arithmetic progression}

Theorem 13. Let $m, n$ and $k$ be integers. Then,

$$
\begin{aligned}
\sum_{j=0}^{k} P_{n+j m, r} x^{j}=\alpha & m^{2}\left\{\frac{3 x-1}{3 x-x^{2}} \sum_{j=0}^{k} T_{j} x^{j}+\frac{1-x^{k}\left(T_{k+1}+x T_{k-1}\right)}{3-x}\right\} \\
& +\left(P_{m, r}+\alpha m n\right)\left\{\frac{x\left(1-x^{k}\right)}{(1-x)^{2}}-\frac{k x^{k+1}}{1-x}\right\}+P_{n, r}\left(\frac{1-x^{k+1}}{1-x}\right) .
\end{aligned}
$$

Proof. Differentiating both sides of the geometric sum (3.4) with respect to $x$ gives

$$
\sum_{j=0}^{k} j x^{j}=-\frac{k x^{k+1}}{(1-x)}+\frac{x\left(1-x^{k}\right)}{(1-x)^{2}}
$$

Multiply through identity (1.26) by $x^{j}$ and sum over $j$, making use of identities (3.4), (3.14) and Theorem 11 with $\alpha=1$ (triangular number) and $m=-1$.

Note that identity (3.14) could also be obtained directly from Theorem 7 with $r=2(\alpha=0)$, since $P_{j, 2}=j$.

Taking limit of the identity of Theorem 13 as $x$ approaches unity gives the sum of the polygonal numbers with indices in arithmetic progression.

Corollary 14. Let $n, m$ and $k$ be integers. Then,

$$
\sum_{j=0}^{k} P_{m j+n, r}=\left(\frac{\alpha m^{2}}{3}(k-1)+P_{m, r}+\alpha m n\right) T_{k}+(k+1) P_{n, r} .
$$

In particular, for the triangular numbers we have

$$
\sum_{j=0}^{k} T_{m j+n}=\left(\frac{m^{2}}{3}(k-1)+T_{m}+m n\right) T_{k}+(k+1) T_{n} .
$$

Using $x=-1$ in Theorem 13 gives the alternating sum of the polygonal numbers with indices in arithmetic progression. 
Corollary 15. Let $n, m$ and $k$ be integers. Then,

$$
\sum_{j=0}^{k}(-1)^{j} P_{m j+n, r}=\left\{\begin{array}{l}
P_{n, r}+\frac{k}{2} P_{m, r}+\frac{\alpha m k}{2}\left(n+\frac{m k}{2}\right), \quad \text { if } k \text { is even; } \\
-\frac{k+1}{4}\left\{2 P_{m, r}+\alpha m(m(k-1)+2 n)\right\}, \quad \text { if } k \text { is odd. }
\end{array}\right.
$$

Dropping terms proportional to $x^{k}$ in Theorem 13 gives the generating function of polygonal numbers with indices in arithmetic progression.

Corollary 16. Let $m$ and $n$ be integers. Then,

$$
\sum_{j=0}^{\infty} P_{m j+n, r} x^{j}=\frac{P_{n, r}}{1-x}+\frac{\left(P_{m, r}+\alpha m n\right) x}{(1-x)^{2}}+\frac{\alpha m^{2} x^{2}}{(1-x)^{3}}, \quad x \neq 1 .
$$

In particular, we have

$$
\begin{gathered}
\sum_{j=0}^{\infty} T_{m j+n} x^{j}=\frac{T_{n}}{1-x}+\frac{\left(T_{m}+m n\right) x}{(1-x)^{2}}+\frac{m^{2} x^{2}}{(1-x)^{3}} \\
\sum_{j=0}^{\infty} P_{j, r} x^{j}=\frac{x}{(1-x)^{2}}+\frac{\alpha x^{2}}{(1-x)^{3}}, \\
\sum_{j=0}^{\infty} T_{j} x^{j}=\frac{x}{(1-x)^{2}}+\frac{x^{2}}{(1-x)^{3}}
\end{gathered}
$$

\section{Quadratic properties: squares and products of polyg- onal numbers}

Here various identities and relations involving products of polygonal numbers will be developed. These will include partial sums $\sum_{j=0}^{k} P_{j, r}^{2} x^{j}, \sum_{j=0}^{k} P_{j+m, r}^{2} x^{j}, \sum_{j=0}^{k} P_{j+a, r} P_{j+b} x^{j}$ and the corresponding generating functions.

Lemma 2. If $j$ is an integer, then,

$$
\begin{aligned}
& 4 P_{j, r} P_{j-1, r}=P_{j, r}^{2}+4 P_{j-1, r}^{2}-P_{j-2, r}^{2}+2 \alpha P_{j-2, r}-\alpha^{2}, \\
& 4 P_{j, r} P_{j-1, r}=4 P_{j, r}^{2}+P_{j-1, r}^{2}-P_{j+1, r}^{2}+2 \alpha P_{j+1, r}-\alpha^{2} .
\end{aligned}
$$

Proof. Square both sides of identity (1.11) and re-arrange to obtain identity (4.1). Square both sides of identity (1.12) and re-arrange to obtain identity (4.2).

Lemma 3. If $j$ is an integer, then,

$$
P_{j+1, r}^{2}-2 P_{j, r}^{2}+P_{j-1, r}^{2}=6 \alpha P_{j, r}+(\alpha-1)^{2}+1
$$


Proof. Square both sides of the recurrence relation

$$
P_{j+1, r}+P_{j-1, r}=2 P_{j, r}+\alpha
$$

and re-arrange to obtain

$$
2 P_{j+1, r} P_{j-1, r}=4 P_{j, r}^{2}-P_{j+1, r}^{2}-P_{j-1, r}^{2}+4 \alpha P_{j, r}+\alpha^{2} .
$$

The exact formula (1.2) gives

$$
P_{j+1, r}-P_{j-1, r}=2+\alpha(2 j-1) .
$$

Squaring both sides of each of (4.3) and (4.5) and subtracting, we find

$$
P_{j+1, r} P_{j-1, r}=P_{j, r}^{2}-\alpha P_{j, r}+\alpha-1 .
$$

Eliminating $P_{j+1, r} P_{j-1, r}$ between (4.4) and (4.6) yields the identity stated in Lemma 3.

Lemma 4. If $j$ is an integer, then,

$$
2 P_{j, r} P_{j+1, r}=P_{j-1, r} P_{j+1, r}+P_{j, r} P_{j+2, r}-(\alpha-1) .
$$

Proof. We have

$$
\begin{aligned}
2 P_{j, r} & P_{j+1, r}-P_{j, r} P_{j+2, r}+\alpha-1 \\
& =P_{j, r}\left(2 P_{j+1, r}-P_{j+2, r}\right)+\alpha-1 \\
& =P_{j, r}\left(P_{j, r}-\alpha\right)+\alpha-1, \quad \text { by }(1.5), \\
& =P_{j, r}^{2}-\alpha P_{j, r}+\alpha-1 \\
& =P_{j-1, r} P_{j+1, r}, \quad \text { by }(4.6) .
\end{aligned}
$$

Lemma 5. Let $a, b$ and $j$ be integers. Then,

$$
\begin{aligned}
P_{j+a, r} P_{j+b, r}=\frac{1}{2} P_{j+a, r}^{2}+ & \frac{1}{2} P_{j+b, r}^{2}-\frac{1}{2} P_{a-b, r}^{2} \\
-\frac{\alpha^{2}}{2}(a-b)^{2}(j+b)^{2}-\alpha(a-b) P_{a-b, r}(j+b) & \\
P_{j+a+1, r} P_{j+b+1, r}-P_{j+a, r} P_{j+b, r}= & \frac{1}{2} P_{j+a+1, r}^{2}+\frac{1}{2} P_{j+b+1, r}^{2}-\frac{1}{2} P_{j+a, r}^{2}-\frac{1}{2} P_{j+b, r}^{2} \\
& -\frac{\alpha^{2}}{2}(a-b)^{2}(2 j+2 b+1)-\alpha(a-b) P_{a-b, r}, \\
P_{j+a+1, r} P_{j+b+1, r}-P_{j+a, r} P_{j+b, r}=\frac{1}{2}\left(\frac{a-b+1}{a-b-1}\right)\left(P_{j+a, r}^{2}-P_{j+b+1, r}^{2}\right) & +\frac{1}{2}\left(\frac{a-b-1}{a-b+1}\right)\left(P_{j+b, r}^{2}-P_{j+a+1, r}^{2}\right), \quad|a-b| \neq 1 .
\end{aligned}
$$


Proof. Set $j=1$ in (1.26) and write $j$ for $n$ :

$$
P_{j+m, r}-P_{j, r}=P_{m, r}+\alpha m j .
$$

squaring both sides and re-arranging gives

$$
2 P_{j+m, r} P_{j, r}=P_{j+m, r}^{2}+P_{j, r}^{2}-P_{m, r}^{2}-2 \alpha m j P_{m, r}-\alpha^{2} m^{2} j^{2} .
$$

Set $j \rightarrow j+b, m \rightarrow a-b$ to obtain identity (4.7).

Shift the index in (4.7) and subtract identity (4.7) from the result. This gives identity (4.8). Arrange identity (2.5) as

$$
T_{m-2} P_{j+m, r}+T_{m-1} P_{j+1, r}=T_{m-2} P_{j, r}+T_{m-1} P_{j+m-1, r},
$$

and set $j \rightarrow j+a$ and $m=b-a+1$ to obtain

$$
T_{b-a-1} P_{j+b+1, r}+T_{b-a} P_{j+a+1, r}=T_{b-a-1} P_{j+a, r}+T_{b-a} P_{j+b, r} .
$$

Squaring both sides of the above relation and rearranging the terms we get

$$
\begin{aligned}
P_{j+a+1, r} P_{j+b+1, r}-P_{j+a, r} P_{j+b, r}= & \frac{1}{2} \frac{T_{a-b}}{T_{a-b-1}}\left(P_{j+a, r}^{2}-P_{j+b+1, r}^{2}\right) \\
& +\frac{1}{2} \frac{T_{a-b-1}}{T_{a-b}}\left(P_{j+b, r}^{2}-P_{j+a+1, r}^{2}\right) ;
\end{aligned}
$$

from which identity (4.9) follows.

Theorem 17. If $j$ and $m$ are integers, then,

$$
T_{m-3}\left(P_{j+m+1, r}^{2}-P_{j, r}^{2}\right)-(m-3)(m+1)\left(P_{j+m, r}^{2}-P_{j+1, r}^{2}\right)+T_{m}\left(P_{j+m-1, r}^{2}-P_{j+2, r}^{2}\right)=0 .
$$

Proof. Write identity (2.5) as

$$
T_{m-2}\left(P_{j+m, r}-P_{j}\right)=T_{m-1}\left(P_{j+m-1}-P_{j+1}\right) .
$$

Square both sides of the above relation and re-arrange the terms, obtaining,

$$
\begin{aligned}
& 2 T_{m-2}^{2} P_{j, r} P_{j+m, r}-2 T_{m-1}^{2} P_{j+1, r} P_{j+m-1, r} \\
& \quad=T_{m-2}^{2}\left(P_{j+m, r}^{2}+P_{j, r}^{2}\right)-T_{m-1}^{2}\left(P_{j+m-1, r}^{2}+P_{j+1, r}^{2}\right) .
\end{aligned}
$$

Write $j+1$ for $j$ in relation (4.11) to get

$$
\begin{aligned}
& 2 T_{m-2}^{2} P_{j+1, r} P_{j+m+1, r}-2 T_{m-1}^{2} P_{j+2, r} P_{j+m, r} \\
& \quad=T_{m-2}^{2}\left(P_{j+m+1, r}^{2}+P_{j+1, r}^{2}\right)-T_{m-1}^{2}\left(P_{j+m, r}^{2}+P_{j+2, r}^{2}\right) .
\end{aligned}
$$

Subtract (4.11) from (4.12) to get

$$
\begin{aligned}
& 2 T_{m-2}^{2}\left(P_{j+1, r} P_{j+m+1, r}-P_{j, r} P_{j+m, r}\right) \\
& -2 T_{m-1}^{2}\left(P_{j+2, r} P_{j+m, r}-P_{j+1, r} P_{j+m-1, r}\right) \\
& \quad=T_{m-2}^{2}\left(P_{j+m+1, r}^{2}+P_{j+1, r}^{2}-P_{j+m, r}^{2}-P_{j, r}^{2}\right) \\
& \quad \quad-T_{m-1}^{2}\left(P_{j+m, r}^{2}+P_{j+2, r}^{2}-P_{j+m-1, r}^{2}-P_{j+1, r}^{2}\right) .
\end{aligned}
$$


Using Lemma 5, identity (4.13) is

$$
\begin{aligned}
& T_{m-2}^{2}\left(\frac{T_{m-1}}{T_{m}}\left(P_{j, r}^{2}-P_{j+m+1, r}^{2}\right)+\frac{T_{m}}{T_{m-1}}\left(P_{j+m, r}^{2}-P_{j+1, r}^{2}\right)\right) \\
& \quad-T_{m-1}^{2}\left(\frac{T_{m-2}}{T_{m-3}}\left(P_{j+m-1, r}^{2}-P_{j+2, r}^{2}\right)+\frac{T_{m-3}}{T_{m-2}}\left(P_{j+1, r}^{2}-P_{j+m, r}^{2}\right)\right) \\
& =T_{m-2}^{2}\left(P_{j+m+1, r}^{2}+P_{j+1, r}^{2}-P_{j+m, r}^{2}-P_{j, r}^{2}\right) \\
& \quad-T_{m-1}^{2}\left(P_{j+m, r}^{2}+P_{j+2, r}^{2}-P_{j+m-1, r}^{2}-P_{j+1, r}^{2}\right) .
\end{aligned}
$$

The identity given in the theorem follows upon clearing fractions and simplification.

Corollary 18. If $j$ is an integer, then,

$$
\begin{gathered}
P_{j, r}^{2}-4 P_{j+1, r}^{2}+5 P_{j+2, r}^{2}-5 P_{j+4, r}^{2}+4 P_{j+5, r}^{2}-P_{j+6, r}^{2}=0, \\
P_{j, r}^{2}-5 P_{j+1, r}^{2}+10 P_{j+2, r}^{2}-10 P_{j+3, r}^{2}+5 P_{j+4, r}^{2}-P_{j+5, r}^{2}=0 \\
2 P_{j, r}^{2}-7 P_{j+1, r}^{2}+7 P_{j+2, r}^{2}-7 P_{j+5, r}^{2}+7 P_{j+6, r}^{2}-2 P_{j+7, r}^{2}=0 .
\end{gathered}
$$

Proof. To prove (4.14) set $m=-3$ or $m=5$ in Theorem 17. Setting $m=4$ or $m=-2$ in Theorem 17 proves (4.15) while (4.16) follows from $m=-4$ in the theorem.

Theorem 19. For any integers $j$ and $m$,

$$
\begin{aligned}
& T_{m-2}\left(P_{j+m+1, r}^{2}-2 P_{j+m, r}^{2}+P_{j+m-1, r}^{2}-P_{j+1, r}^{2}+2 P_{j, r}^{2}-P_{j-1, r}^{2}\right) \\
& \quad-T_{m-1}\left(P_{j+m, r}^{2}-2 P_{j+m-1, r}^{2}+P_{j+m-2, r}^{2}-P_{j+2, r}^{2}+2 P_{j+1, r}^{2}-P_{j, r}^{2}\right)=0 .
\end{aligned}
$$

Proof. Multiply through identity (4.10) by $6 \alpha$ to obtain

$$
T_{m-2}\left(6 \alpha P_{j+m, r}-6 \alpha P_{j, r}\right)-T_{m-1}\left(6 \alpha P_{j+m-1, r}-6 \alpha P_{j+1, r}\right)=0 .
$$

Now use the identity given in Lemma 3 as

$$
6 \alpha P_{j, r}=P_{j+1, r}^{2}-2 P_{j, r}^{2}+P_{j-1, r}^{2}-(\alpha-1)^{2}-1 .
$$

For a general identity relating the pure powers of the polygonal numbers see Theorem 42 .

Theorem 20. Let $m$ and $j$ be integers. Then,

$$
\begin{aligned}
& m(m-2) P_{j+m+1, r}^{2}-2\left(m^{2}-4\right) P_{j+m, r}^{2}+m(m+2) P_{j+m-1, r}^{2} \\
&= m(m+2) P_{j+1, r}^{2}-2\left(m^{2}-4\right) P_{j, r}^{2} \\
&+m(m-2)\left(P_{j-1, r}^{2}+2 \alpha P_{j+m+1, r}-2 \alpha P_{j-1, r}\right) .
\end{aligned}
$$

Proof. Arrange identity (2.5) as

$$
T_{m-2} P_{j+m, r}-T_{m-1} P_{j+m-1, r}=T_{m-2} P_{j, r}-T_{m-1} P_{j+1, r}
$$


Square both sides of the above identity and use identities (4.1) and (4.2) to eliminate the products $P_{j+m, r} P_{j+m-1, r}$ and $P_{j, r} P_{j+1, r}$. The result is

$$
\begin{aligned}
& T_{m-1} T_{m-2} P_{j+m+1, r}^{2}+\left(2 T_{m-2}^{2}-4 T_{m-1} T_{m-2}\right) P_{j+m, r}^{2}+\left(2 T_{m-1}^{2}-T_{m-1} T_{m-2}\right) P_{j+m-1, r}^{2} \\
& =\left(2 T_{m-1}^{2}-T_{m-1} T_{m-2}\right) P_{j+1, r}^{2}+\left(2 T_{m-2}^{2}-4 T_{m-1} T_{m-2}\right) P_{j, r}^{2}+T_{m-1} T_{m-2} P_{j-1, r}^{2} \\
& \quad+2 \alpha T_{m-1} T_{m-2}\left(P_{j+m+1, r}-P_{j-1, r}\right)
\end{aligned}
$$

from which the identity of the theorem follows upon substituting for the triangular numbers.

We have the following examples:

$$
\begin{gathered}
P_{j+3, r}^{2}-3 P_{j+2, r}^{2}+3 P_{j+1, r}^{2}-P_{j, r}^{2}=2 \alpha\left(P_{j+3, r}-P_{j, r}\right), \\
3 P_{j+2, r}^{2}-3 P_{j+1, r}^{2}+P_{j, r}^{2}-P_{j+3, r}^{2}=6 \alpha\left(P_{j+1, r}-P_{j+2, r}\right) .
\end{gathered}
$$

Identities (4.18) and (4.19) come from evaluating the identity stated in Theorem 20 at $m=1$ and $m=-1$, respectively.

\subsection{Partial sums and generating functions}

Theorem 21. If $k$ is an integer, then,

$$
2 \sum_{j=0}^{k}(-1)^{j} P_{j, r} P_{j+1, r}=\left\{\begin{array}{l}
P_{k, r} P_{k+2, r}, \quad \text { if } k \text { is even } \\
\alpha-1-P_{k, r} P_{k+2, r}, \quad \text { if } k \text { is odd } .
\end{array}\right.
$$

Proof. Multiply through the identity stated in Lemma 4 by $(-1)^{j}$ and sum over $j$ :

$$
\begin{aligned}
2 \sum_{j=0}^{k}(-1)^{j} P_{j, r} P_{j+1, r}= & \sum_{j=0}^{k}(-1)^{j}\left(P_{j-1, r} P_{j+1, r}+P_{j, r} P_{j+2, r}\right) \\
& -(\alpha-1) \sum_{j=0}^{k}(-1)^{j} .
\end{aligned}
$$

Use summation formula (1.44) with $f_{j}=P_{j-1, r} P_{j+1, r}$ to evaluate the first sum on the right hand side.

Theorem 22. If $k$ is an integer, then,

$$
\begin{aligned}
\sum_{j=0}^{k} P_{j, r}^{2} x^{j}= & \frac{6 \alpha x}{(1-x)^{2}}\left(\frac{x}{(1-x)^{2}}+\frac{\alpha x^{2}\left(1-x^{k}\right)}{(1-x)^{3}}-\frac{x^{k+1}\left(P_{k+1, r}-x P_{k, r}\right)}{(1-x)^{2}}\right) \\
& +\frac{\left((\alpha-1)^{2}+1\right) x\left(1-x^{k+1}\right)}{(1-x)^{3}}-\frac{x^{k+1}\left(P_{k+1, r}^{2}-x P_{k, r}^{2}\right)}{(1-x)^{2}}-\frac{(\alpha-1)^{2} x}{(1-x)^{2}} .
\end{aligned}
$$

Proof. Multiply through the identity of Lemma 3 and sum over $j$ to obtain

$$
\begin{aligned}
& \sum_{j=0}^{k} P_{j+1, r}^{2} x^{j}-2 \sum_{j=0}^{k} P_{j, r}^{2} x^{j}+\sum_{j=0}^{k} P_{j-1, r}^{2} x^{j} \\
& =6 \alpha \sum_{j=0}^{k} P_{j, r} x^{j}+\left((\alpha-1)^{2}+1\right) \sum_{j=0}^{k} x^{j}
\end{aligned}
$$


which, by shifting the index in each sum appropriately and re-arranging can be written

$$
\begin{array}{r}
(1-x)^{2} \sum_{j=0}^{k} P_{j, r}^{2} x^{j}=6 \\
\alpha x \sum_{j=0}^{k} P_{j, r} x^{j}+\left((\alpha-1)^{2}+1\right) x \sum_{j=0}^{k} x^{j} \\
-x^{k+1} P_{k+1, r}^{2}+x^{k+2} P_{k, r}^{2}-(\alpha-1)^{2} x ;
\end{array}
$$

from which the result follows when we use Theorem 7.

Corollary 23. If $k$ is an integer, then,

$$
\begin{aligned}
& \sum_{j=0}^{k} P_{j, r}^{2}=\left(\left\{(k+3) \frac{\alpha}{5}+P_{k, r}-P_{k+1, r}\right\} \alpha T_{k-1}+\frac{1}{3} k P_{k-1, r}^{2}-\frac{1}{3}(2 k-3) P_{k, r}^{2}\right) T_{k+1} \\
& +\frac{1}{3}(k+1) T_{k-1} P_{k+1, r}^{2}, \\
& \sum_{j=0}^{k}(-1)^{j} P_{j, r}^{2}=\left\{\begin{array}{l}
\frac{\alpha}{8}\left(\alpha-\left\{3\left(P_{k+1, r}-P_{k, r}\right)-1\right\}\right)+\frac{1}{8}\left(P_{k+1, r}^{2}-P_{k-1, r}^{2}+4 P_{k, r}^{2}\right), \quad \text { if } k \text { is even } ; \\
\frac{\alpha}{8}\left(-2 \alpha+\left\{3\left(P_{k+1, r}-P_{k, r}\right)+1\right\}\right)-\frac{1}{8}\left(P_{k+1, r}^{2}-P_{k-1, r}^{2}+4 P_{k, r}^{2}\right), \quad \text { if } k \text { is odd. }
\end{array}\right.
\end{aligned}
$$

Proof. Repeated application of L'Hospital's rule to the identity of Theorem 22 at $x=1$ yields identity (4.20); while (4.21) comes from evaluating the identity at $x=-1$.

Corollary 24. We have

$$
\sum_{j=0}^{\infty} P_{j, r}^{2} x^{j}=\frac{6 \alpha x}{(1-x)^{2}}\left\{\frac{x}{(1-x)^{2}}+\frac{\alpha x^{2}}{(1-x)^{3}}\right\}+\frac{\left\{(\alpha-1)^{2}+1\right\} x}{(1-x)^{3}}-\frac{(\alpha-1)^{2} x}{(1-x)^{2}} .
$$

For the triangular numbers, $\alpha=1$ and we have the generating function of the squares of triangular numbers to be given by

$$
\sum_{j=0}^{\infty} T_{j}^{2} x^{j}=\frac{6 x^{2}}{(1-x)^{5}}+\frac{x}{(1-x)^{3}} .
$$

Theorem 25. If $k$ is an integer, then,

$$
\begin{aligned}
\sum_{j=0}^{k} P_{j, r}^{2} x^{j}= & \frac{(\alpha-1)^{2} x^{4}-\{1+2 \alpha(1-2 \alpha)\} x^{3}+\{\alpha(\alpha+4)-1\} x^{2}+x}{(1-x)^{5}} \\
- & \frac{\left((1-x)^{5}+x^{5}\right)}{(1-x)^{5}} P_{k+1, r}^{2} x^{k+1}-\frac{\left((1-x)^{5}+x^{5}-5 x^{4}\right)}{(1-x)^{5}} P_{k+2, r}^{2} x^{k+2} \\
& -\frac{\left(1-5 x+10 x^{2}\right)}{(1-x)^{5}} P_{k+3, r}^{2} x^{k+3}-\frac{(1-5 x)}{(1-x)^{5}} P_{k+4, r}^{2} x^{k+4}-\frac{P_{k+5, r}^{2} x^{k+5}}{(1-x)^{5}}
\end{aligned}
$$

Proof. Write recurrence relation (4.15) as

$$
P_{j, r}^{2}=5 P_{j+1, r}^{2}-10 P_{j+2, r}^{2}+10 P_{j+3, r}^{2}-5 P_{j+4, r}^{2}+P_{j+5, r}^{2} .
$$

In Lemma 1 choose $\left(f_{1}, f_{2}, f_{3}, f_{4}, f_{5}\right)=(5,-10,10,-5,1),\left(c_{1}, c_{2}, c_{3}, c_{4}, c_{5}\right)=(-1,-2,-3,-4,-5)$ and $\left(X_{j}\right)=\left(P_{j, r}^{2}\right)$. 
Corollary 26. We have

$$
\sum_{j=0}^{\infty} P_{j, r}^{2} x^{j}=\frac{(\alpha-1)^{2} x^{4}-\{1+2 \alpha(1-2 \alpha)\} x^{3}+\{\alpha(\alpha+4)-1\} x^{2}+x}{(1-x)^{5}}
$$

Theorem 27. Let $m$ and $k$ be integers. Then,

$$
\begin{aligned}
\sum_{j=0}^{k} P_{j+m, r}^{2} x^{j}=\frac{m(m-2) x^{2}-2\left(m^{2}-4\right) x+m(m+2)}{m(m+2) x^{2}-2\left(m^{2}-4\right) x+m(m-2)} \sum_{j=0}^{k} P_{j, r}^{2} x^{j} \\
-\frac{2 \alpha m(m-2)\left(x^{2} \sum_{j=0}^{k} P_{j, r} x^{j}-\sum_{j=0}^{k} P_{j+m, r} x^{j}\right)}{m(m+2) x^{2}-2\left(m^{2}-4\right) x+m(m-2)} \\
+\frac{2 \alpha m(m-2)\left(x^{k+2} P_{k, r}+x^{k+1} P_{k+m+1, r}-x P_{-1, r}-P_{m, r}\right)}{m(m+2) x^{2}-2\left(m^{2}-4\right) x+m(m-2)} \\
+\frac{m(m+2)\left(x^{k+2} P_{k+m, r}^{2}+P_{k+1, r}^{2} x^{k+1}-x P_{m-1, r}^{2}\right)}{m(m+2) x^{2}-2\left(m^{2}-4\right) x+m(m-2)} \\
\quad-\frac{m(m-2)\left(x^{k+2} P_{k, r}^{2}+x^{k+1} P_{k+m+1, r}^{2}-x P_{-1, r}^{2}-P_{m, r}^{2}\right)}{m(m+2) x^{2}-2\left(m^{2}-4\right) x+m(m-2)} .
\end{aligned}
$$

Proof. Multiply through the identity of Theorem 20 by $x^{j}$ and sum over $j$, shifting the index in each sum as appropriate.

Corollary 28. Let $m$ and $k$ be integers. Then,

$$
\begin{aligned}
\sum_{j=0}^{k} P_{j+m, r}^{2}= & \frac{m(m-2)}{8}\left\{\left(P_{m, r}-\alpha\right)^{2}-\left(P_{k+m+1, r}-\alpha\right)^{2}-\left(P_{k, r}-\alpha\right)^{2}+1\right\} \\
& +\frac{\alpha m^{2}(m-2)}{8}\left\{P_{k+m, r}+P_{k+1, r}-P_{m-1, r}\right\}+\frac{m(m+2)}{8}\left(P_{k+m, r}^{2}+P_{k+1, r}^{2}-P_{m-1, r}^{2}\right) \\
& +\left(\left\{(k+3) \frac{\alpha}{5}+P_{k, r}-P_{k+1, r}\right\} \alpha T_{k-1}+\frac{1}{3} k P_{k-1, r}^{2}-\frac{1}{3}(2 k-3) P_{k, r}^{2}\right) T_{k+1} \\
& +\frac{1}{3}(k+1) T_{k-1} P_{k+1, r}^{2} .
\end{aligned}
$$

Proof. Set $x=1$ in Theorem 27 making use of (3.6), (3.12) and (4.20).

Theorem 29. Let $m$ and $k$ be integers. Then,

$$
\begin{aligned}
& \sum_{j=0}^{k} P_{j+m, r}^{2} x^{j}=\frac{x^{2} T_{m-3}-(m-3)(m+1) x+T_{m}}{x T_{m-3}-(m-3)(m+1) x^{2}+T_{m} x^{3}} \sum_{j=0}^{k} P_{j, r}^{2} x^{j} \\
& +\frac{T_{m} x^{k+1} P_{k+2, r}^{2}+\left(T_{m}-(m-3)(m+1) x\right) x^{k} P_{k+1, r}^{2}}{T_{m-3}-(m-3)(m+1) x+T_{m} x^{2}} \\
& +\frac{T_{m} x^{k+2} P_{k+m, r}^{2}-T_{m-3} x^{k+1} P_{k+m+1, r}^{2}}{T_{m-3}-(m-3)(m+1) x+T_{m} x^{2}} \\
& -\frac{T_{m}+x T_{m} P_{m-1, r}^{2}-T_{m-3} P_{m, r}^{2}}{T_{m-3}-(m-3)(m+1) x^{2}+T_{m} x^{2}} \text {. }
\end{aligned}
$$


Proof. Multiply through the identity of Theorem 17 by $x^{j}$ and sum over $j$, shifting the index in each sum as appropriate.

Theorem 30. Let $a, b$ and $k$ be integers such that $\mid a-b>1$. Let $x \in \mathbb{C}, x \neq 1$. Then,

$$
\begin{aligned}
2 \sum_{j=0}^{k} P_{j+a} P_{j+b} x^{j}=- & \frac{\left(T_{b-a}^{2}-x T_{b-a-1}^{2}\right)}{T_{b-a} T_{b-a-1}(1-x)} \sum_{j=0}^{k} P_{j+a}^{2} x^{j}-\frac{\left(T_{b-a-1}^{2}-x T_{b-a}^{2}\right)}{T_{b-a} T_{b-a-1}(1-x)} \sum_{j=0}^{k} P_{j+b}^{2} x^{j} \\
& +\frac{T_{b-a}}{T_{b-a-1}} \frac{\left(P_{a}^{2}-x^{k+1} P_{k+a+1}^{2}\right)}{(1-x)}+\frac{T_{b-a-1}}{T_{b-a}} \frac{\left(P_{b}^{2}-x^{k+1} P_{k+b+1}^{2}\right)}{(1-x)} \\
& -2 \frac{x^{k+1}}{(1-x)} P_{k+a+1} P_{k+b+1}+2 \frac{P_{a} P_{b}}{(1-x)} .
\end{aligned}
$$

Proof. Multiply through identity (4.9) by $x^{j}$ and sum over $j$, shifting the index in each sum as appropriate.

Corollary 31. Let $a, b$ and $k$ be integers. Then,

$$
\begin{aligned}
2 \sum_{j=0}^{k} P_{j+a, r} P_{j+b, r}= & \frac{a(a-2)}{8}\left\{\left(P_{a, r}-\alpha\right)^{2}-\left(P_{k+a+1, r}-\alpha\right)^{2}-\left(P_{k, r}-\alpha\right)^{2}+1\right\} \\
& +\frac{b(b-2)}{8}\left\{\left(P_{b, r}-\alpha\right)^{2}-\left(P_{k+b+1, r}-\alpha\right)^{2}-\left(P_{k, r}-\alpha\right)^{2}+1\right\} \\
& +\frac{a(a+2)}{8}\left(P_{k+a, r}^{2}+P_{k+1, r}^{2}-P_{a-1, r}^{2}\right)+\frac{b(b+2)}{8}\left(P_{k+b, r}^{2}+P_{k+1, r}^{2}-P_{b-1, r}^{2}\right) \\
& +\frac{\alpha a^{2}(a-2)}{8}\left\{P_{k+a, r}+P_{k+1, r}-P_{a-1, r}\right\}+\frac{\alpha b^{2}(b-2)}{8}\left\{P_{k+b, r}+P_{k+1, r}-P_{b-1, r}\right\} \\
& +2 T_{k+1}\left(\left\{(k+3) \frac{\alpha}{5}+P_{k, r}-P_{k+1, r}\right\} \alpha T_{k-1}+\frac{1}{3} k P_{k-1, r}^{2}-\frac{1}{3}(2 k-3) P_{k, r}^{2}\right) \\
& +(k+1)\left\{\frac{2}{3} T_{k-1} P_{k+1, r}^{2}-P_{a-b, r}\left\{P_{a-b, r}+\alpha(a-b)(k+2 b)\right\}\right\} \\
& -(k+1) \frac{\alpha^{2}(a-b)^{2}}{6}\left(2 k^{2}+k+6 k b+6 b^{2}\right) .
\end{aligned}
$$

Proof. Sum each term in identity (4.7) from 0 to $k$, making use of Corollary 28.

\section{Sums and identities involving reciprocals}

Identity (2.5) at $j=0$ invokes a summation identity.

Theorem 32. For any integer $k$,

$$
\sum_{j=1}^{k} \frac{T_{j}}{P_{j, r} P_{j+1, r}}=\frac{T_{k}}{P_{k+1, r}}=\frac{T_{k}}{T_{k+1}+(\alpha-1) T_{k}}
$$

Proof. Setting $j=0$ in (2.5), writing $j+1$ for $m$, we have

$$
T_{j} P_{j, r}-T_{j-1} P_{j+1, r}=T_{j}
$$


Dividing through by $P_{j, r} P_{j+1, r}$,

$$
\frac{T_{j}}{P_{j+1, r}}-\frac{T_{j-1}}{P_{j}}=\frac{T_{j}}{P_{j+1, r} P_{j, r}},
$$

from which the result follows by telescoping summation.

In particular, we have

$$
\sum_{j=1}^{k} \frac{1}{T_{j+1}}=\frac{T_{k}}{T_{k+1}}=\frac{k}{k+2} .
$$

Corollary 33. For $\alpha \neq 0$ (that is $r \neq 2$ ),

$$
\sum_{j=1}^{\infty} \frac{T_{j}}{P_{j, r} P_{j+1, r}}=\frac{1}{\alpha} .
$$

Proof. We have

$$
\sum_{j=1}^{\infty} \frac{T_{j}}{P_{j, r} P_{j+1, r}}=\lim _{k \rightarrow \infty} \frac{T_{k}}{T_{k+1}+(\alpha-1) T_{k}}=\lim _{k \rightarrow \infty} \frac{1}{T_{k+1} / T_{k}+(\alpha-1)}=\frac{1}{\alpha} .
$$

Theorem 34 and Theorem 38 are both consequences of identity (1.24).

Theorem 34. If $k$ and $n$ are non-negative integers, then,

$$
\begin{aligned}
\alpha n^{2} \sum_{j=1}^{k} \frac{1}{P_{j+n, r}} & -\left(T_{n-1} T_{n} \alpha^{2}+(\alpha-1) n^{2}\right) \sum_{j=1}^{k} \frac{1}{P_{j, r} P_{j+n, r}} \\
& =\sum_{j=1}^{n}\left(\frac{P_{j+k-n, r}}{P_{j+k, r}}-\frac{P_{j-n, r}}{P_{j, r}}\right) .
\end{aligned}
$$

Proof. Identity (1.24) written out and re-arranged is

$$
P_{j, r}^{2}-P_{j+n, r} P_{j-n, r}=\left(P_{n, r}+P_{-n, r}\right) P_{j, r}-P_{n, r} P_{-n, r} .
$$

Dividing through by $P_{j+n, r} P_{j, r}$ gives

$$
\begin{aligned}
\frac{P_{j, r}}{P_{j+n, r}}-\frac{P_{j-n, r}}{P_{j, r}} & =\frac{P_{n, r}+P_{-n, r}}{P_{j+n, r}}-\frac{P_{n, r} P_{-n, r}}{P_{j, r} P_{j+n, r}} \\
& =\frac{\alpha n^{2}}{P_{j+n, r}}-\frac{\left(T_{n-1} T_{n} \alpha^{2}+(\alpha-1) n^{2}\right)}{P_{j, r} P_{j+n, r}}
\end{aligned}
$$

where we have used (1.3), (1.8) and (1.10). The result follows from summing over $j$ and making use of (1.47), with $f_{j}=P_{j-n, r} / P_{j, r}$.

Corollary 35. If $k$ is a non-negative integer, then,

$$
\alpha \sum_{j=1}^{k} \frac{1}{P_{j+1, r}}-(\alpha-1) \sum_{j=1}^{k} \frac{1}{P_{j, r} P_{j+1, r}}=\frac{P_{k, r}}{P_{k+1, r}} .
$$


Corollary 36. We have

$$
\alpha \sum_{j=1}^{\infty} \frac{1}{P_{j+1, r}}-(\alpha-1) \sum_{j=1}^{\infty} \frac{1}{P_{j, r} P_{j+1, r}}=1 .
$$

Theorem 37. If $k$ is a non-negative integer, then,

$$
\sum_{j=1}^{k}(-1)^{j-1} \frac{2 P_{j+1, r} P_{j+2, r}+\alpha-1}{P_{j, r} P_{j+1, r} P_{j+2, r} P_{j+3, r}}=\frac{1}{3(\alpha+1)}+\frac{(-1)^{k-1}}{P_{k+1, r} P_{k+3, r}} .
$$

Proof. The identity given in Lemma 4, with a shift of the index, allows us to write

$$
\frac{1}{P_{j, r} P_{j+2, r}}+\frac{1}{P_{j+1, r} P_{j+3, r}}=\frac{2 P_{j+1, r} P_{j+2, r}+\alpha-1}{P_{j, r} P_{j+1, r} P_{j+2, r} P_{j+3, r}} ;
$$

from which the result follows by telescoping summation using (1.46) with $f_{j}=1 /\left(P_{j, r} P_{j+2, r}\right)$.

For the triangular numbers, $(\alpha=1)$, we have

$$
2 \sum_{j=1}^{k} \frac{(-1)^{j-1}}{T_{j} T_{j+3}}=\frac{1}{6}+\frac{(-1)^{k-1}}{T_{k+1} T_{k+3}} .
$$

Theorem 38. Let $m, n$ and $k$ be integers. Then,

$$
\begin{aligned}
\sum_{j=0}^{k} \frac{P_{m j+n, r} P_{m j-n, r}}{P_{m j, r}-P_{-n, r}} x^{j}=\alpha & m^{2}\left\{\frac{3 x-1}{3 x-x^{2}} \sum_{j=0}^{k} T_{j} x^{j}+\frac{1-x^{k}\left(T_{k+1}+x T_{k-1}\right)}{3-x}\right\} \\
+ & P_{m, r}\left\{\frac{x\left(1-x^{k}\right)}{(1-x)^{2}}-\frac{k x^{k+1}}{1-x}\right\}-P_{n, r}\left(\frac{1-x^{k+1}}{1-x}\right) .
\end{aligned}
$$

Proof. Identity (1.24) is

$$
\frac{P_{m j+n, r} P_{m j-n, r}}{P_{m j, r}-P_{-n, r}}=P_{m j, r}-P_{n, r} .
$$

Multiply both sides by $x^{j}$ and sum over $j$, making use of Theorem 13 with $n=0$.

Corollary 39. If $k$ and $m$ are integers, then,

$$
\sum_{j=0}^{k} \frac{P_{m j+1, r} P_{m j-1, r}}{P_{m j, r}-\alpha+1}=\left(\frac{(k-1)}{3} m^{2} \alpha+P_{m, r}\right) T_{k}-k-1 .
$$

Proof. Set $x=1, n=1$ in the identity given in Theorem 38 and make use of identity (1.17).

In particular, we have

$$
\sum_{j=1}^{k} \frac{T_{m j+1} T_{m j-1}}{T_{m j}}=\left(\frac{(k-1)}{3} m^{2}+T_{m}\right) T_{k}-k
$$

with the special value involving three consecutive triangular numbers,

$$
\sum_{j=1}^{k} \frac{T_{j+1} T_{j-1}}{T_{j}}=\frac{(k+2)}{3} T_{k}-k
$$




\section{Powers of polygonal numbers}

We have seen that, identity (1.13),

$$
P_{j, r}-3 P_{j+1, r}+3 P_{j+2, r}-P_{j+3, r}=0,
$$

and that, identity (4.15),

$$
P_{j, r}^{2}-5 P_{j+1, r}^{2}+10 P_{j+2, r}^{2}-10 P_{j+3, r}^{2}+5 P_{j+4, r}^{2}-P_{j+5, r}^{2}=0 .
$$

More generally, on account of Euler's finite difference theorem, Gould [7, identity (10.1)],

$$
\sum_{s=0}^{t}(-1)^{t+s}\left(\begin{array}{l}
t \\
s
\end{array}\right) s^{m}=\left\{\begin{array}{l}
0, \quad \text { if } 0 \leq m<t \\
(-1)^{t} t !, \quad \text { if } m=t
\end{array}\right.
$$

we have the results stated in Theorem 40 and Theorem 41.

Theorem 40. Let $n$ be a non-negative integer and $j$ any integer. Then,

$$
\sum_{s=0}^{2 n+1}(-1)^{s}\left(\begin{array}{c}
2 n+1 \\
s
\end{array}\right) P_{j+s, r}^{n}=0 .
$$

Here are some explicit examples:

$$
\begin{gathered}
P_{j, r}^{3}-7 P_{j+1, r}^{3}+21 P_{j+2, r}^{3}-35 P_{j+3, r}^{3}+53 P_{j+4, r}^{3} \\
-21 P_{j+5, r}^{3}+7 P_{j+6, r}^{3}-P_{j+7}^{3}=0, \\
P_{j, r}^{4}-9 P_{j+1, r}^{4}+36 P_{j+2, r}^{4}-84 P_{j+3, r}^{4}+126 P_{j+4, r}^{4}-126 P_{j+5, r}^{4} \\
+84 P_{j+6, r}^{4}-36 P_{j+7, r}^{4}+9 P_{j+8, r}^{4}-P_{j+9, r}^{4}=0, \\
P_{j, r}^{5}-11 P_{j+1, r}^{5}+55 P_{j+2, r}^{5}-165 P_{j+3, r}^{5}+330 P_{j+4, r}^{5}-462 P_{j+5, r}^{5} \\
+462 P_{j+6, r}^{5}-330 P_{j+7, r}^{5}+165 P_{j+8, r}^{5}-55 P_{j+9, r}^{5}+11 P_{j+10, r}^{5}-P_{j+11, r}^{5}=0 .
\end{gathered}
$$

Theorem 41. For $n$ a non-negative integer, let

$$
a(n)=\sum_{s=0}^{2 n}(-1)^{s}\left(\begin{array}{c}
2 n \\
s
\end{array}\right)\left(P_{n-s+1, r}^{n+1}-P_{n-s, r}^{n+1}\right), \quad b(n)=\sum_{s=0}^{2 n}(-1)^{s}\left(\begin{array}{c}
2 n \\
s
\end{array}\right) P_{n-s, r}^{n+1} .
$$

If $j$ is any integer, then,

$$
\sum_{s=0}^{2 n}(-1)^{s}\left(\begin{array}{c}
2 n \\
s
\end{array}\right) P_{j+s, r}^{n+1}=a(n) P_{j+n, r}+b(n) .
$$

Here are a few more explicit examples:

$$
\begin{gathered}
P_{j, r}^{2}-2 P_{j+1, r}^{2}+P_{j+2, r}^{2}=6 \alpha P_{j+1, r}+(\alpha-1)^{2}+1, \\
P_{j, r}^{3}-4 P_{j+1, r}^{3}+6 P_{j+2, r}^{3}-4 P_{j+3, r}^{3}+P_{j+4, r}^{3} \\
=90 \alpha^{2} P_{j+2, r}+24 \alpha^{3}-36 \alpha(\alpha-1), \\
P_{j, r}^{4}-6 P_{j+1, r}^{4}+15 P_{j+2, r}^{4}-20 P_{j+3, r}^{4}+15 P_{j+4, r}^{4}-6 P_{j+5, r}^{4}+P_{j+6, r}^{4} \\
=2520 \alpha^{3} P_{j+3, r}-1080 \alpha^{2}(\alpha-1)+900 \alpha^{4} .
\end{gathered}
$$


Theorem 42. Let $n$ be a non-negative integer and $m$ and $j$ any integers. Then,

$$
\begin{aligned}
T_{m-2} & \sum_{s=0}^{2 n}(-1)^{s}\left(\begin{array}{c}
2 n \\
s
\end{array}\right)\left(P_{j+m-n+s, r}^{n+1}-P_{j-n+s, r}^{n+1}\right) \\
& -T_{m-1} \sum_{s=0}^{2 n}(-1)^{s}\left(\begin{array}{c}
2 n \\
s
\end{array}\right)\left(P_{j+m-n+s-1, r}^{n+1}-P_{j-n+s+1, r}^{n+1}\right)=0 .
\end{aligned}
$$

Proof. Multiply through identity (3.11) by the $a(n)$ defined in Theorem 41 to obtain

$$
\begin{aligned}
& T_{m-2}\left(a(n) P_{j+m, r}\right)-T_{m-2}\left(a(n) P_{j, r}\right) \\
& \quad-T_{m-1}\left(a(n) P_{j+m-1, r}\right)+T_{m-1}\left(a(n) P_{j+1, r}\right)=0 .
\end{aligned}
$$

Now use identity (6.5) in the form

$$
a(n) P_{j, r}=\sum_{s=0}^{2 n}(-1)^{s}\left(\begin{array}{c}
2 n \\
s
\end{array}\right) P_{j+s-n, r}^{n+1}-b(n) .
$$

Here is an example evaluation at $m=-1, n=5$.

$$
\begin{aligned}
P_{j+6, r}^{6}- & 13 P_{j+5, r}^{6}+78 P_{j+4, r}^{6}-286 P_{j+3, r}^{6}+715 P_{j+2, r}^{6}-1287 P_{j+1, r}^{6} \\
+ & 1716 P_{j, r}^{6}-1716 P_{j-1, r}^{6}+1287 P_{j-2, r}^{6}-715 P_{j-3, r}^{6}+286 P_{j-4, r}^{6} \\
& -78 P_{j-5, r}^{6}+13 P_{j-6, r}^{6}-P_{j-7, r}^{6}=0 .
\end{aligned}
$$

\subsection{Partial sums and generating functions}

Theorem 43. Let $n$ be a non-negative integer and $k$ any integer. Then,

$$
\begin{aligned}
\sum_{j=0}^{k} P_{j, r}^{n} x^{j}= & \frac{1}{(1-x)^{2 n+1}} \sum_{s=0}^{2 n+1}\left\{(-1)^{s}\left(\begin{array}{c}
2 n+1 \\
s
\end{array}\right) x^{2 n-s+1} \sum_{j=k+1}^{k+s} P_{j, r}^{n} x^{j}\right\} \\
& -\frac{1}{(1-x)^{2 n+1}} \sum_{s=0}^{2 n+1}\left\{(-1)^{s}\left(\begin{array}{c}
2 n+1 \\
s
\end{array}\right) x^{2 n-s+1} \sum_{j=0}^{s-1} P_{j, r}^{n} x^{j}\right\} .
\end{aligned}
$$

Proof. Multiply through the identity of Theorem 40 by $x^{j}$ and sum,

$$
\sum_{s=0}^{2 n+1}\left\{(-1)^{s}\left(\begin{array}{c}
2 n+1 \\
s
\end{array}\right) \sum_{j=0}^{k} P_{j+s, r}^{n} x^{j}\right\}=0 .
$$

Using (1.40), this is

$$
\sum_{s=0}^{2 n+1}\left\{(-1)^{s}\left(\begin{array}{c}
2 n+1 \\
s
\end{array}\right)\left(x^{-s} \sum_{j=0}^{k} P_{j, r}^{n} x^{j}-x^{-s} \sum_{j=0}^{s-1} P_{j, r}^{n} x^{j}+x^{-s} \sum_{j=k+1}^{k+s} P_{j+s, r}^{n} x^{j}\right)\right\}=0 .
$$

Clearing brackets and multiplying through by $x^{2 n+1}$, we have

$$
\begin{aligned}
\sum_{j=0}^{k} P_{j, r}^{n} x^{j} \sum_{s=0}^{2 n+1}(-1)^{s}\left(\begin{array}{c}
2 n+1 \\
s
\end{array}\right) x^{2 n-s+1}-\sum_{s=0}^{2 n+1}(-1)^{s}\left(\begin{array}{c}
2 n+1 \\
s
\end{array}\right) x^{2 n-s+1} \sum_{j=0}^{s-1} P_{j, r}^{n} x^{j} \\
+\sum_{s=0}^{2 n+1}(-1)^{s}\left(\begin{array}{c}
2 n+1 \\
s
\end{array}\right) x^{2 n-s+1} \sum_{j=k+1}^{k+s} P_{j, r}^{n} x^{j}=0 .
\end{aligned}
$$


The result now follows upon making $\sum_{j=0}^{k} P_{j, r}^{n} x^{j}$ subject in the above equation. Note that

$$
\sum_{s=0}^{2 n+1}(-1)^{s}\left(\begin{array}{c}
2 n+1 \\
s
\end{array}\right) x^{2 n-s+1}=-(1-x)^{2 n+1} \text {. }
$$

Observe that Theorem 10 and Theorem 25 are special cases of Theorem 43.

Taking limit as $k$ approaches infinity in Theorem 43, we obtain the generating function of the powers of polygonal numbers as stated in the next result.

Corollary 44. Let $n$ be a non-negative integer. Then,

$$
\sum_{j=0}^{\infty} P_{j, r}^{n} x^{j}=\frac{1}{(1-x)^{2 n+1}} \sum_{s=1}^{2 n+1}\left\{(-1)^{s-1}\left(\begin{array}{c}
2 n+1 \\
s
\end{array}\right) x^{2 n-s+1} \sum_{j=0}^{s-1} P_{j, r}^{n} x^{j}\right\} .
$$

\section{Weighted sums}

In this section we present some weighted ordinary and binomial summation identities involving the polygonal numbers.

The general summation identities which we require for this purpose are given in Lemmata 6 and 7 and can be proved by induction (see Adegoke [1]).

\subsection{Ordinary summation identities}

Lemma 6. Let $\left(X_{n}\right)$ be any arbitrary sequence. Let $X_{n}, n \in \mathbb{Z}$, satisfy a recurrence relation $X_{n}=f_{1} X_{n-c}+f_{2} X_{n-d}+\lambda$, where $f_{1}$ and $f_{2}$ are non-vanishing complex functions, not dependent on $n$, and $c$ and $d$ are integers. Then,

$$
\begin{gathered}
f_{2} \sum_{j=0}^{k} f_{1}^{j} X_{n-d-j c}=X_{n}-f_{1}^{k+1} X_{n-(k+1) c}-\frac{\lambda\left(1-f_{1}^{k+1}\right)}{1-f_{1}}, \\
f_{1} \sum_{j=0}^{k} f_{2}^{j} X_{n-c-j d}=X_{n}-f_{2}^{k+1} X_{n-(k+1) d}-\frac{\lambda\left(1-f_{2}^{k+1}\right)}{1-f_{2}}, \\
\sum_{j=0}^{k}(-1)^{j} f_{1}^{k-j} f_{2}^{j} X_{n+c+(c-d) j} \\
=f_{1}^{k+1} X_{n}+(-1)^{k} f_{2}^{k+1} X_{n+(c-d)(k+1)}+\frac{\lambda\left(f_{1}^{k+1}+(-1)^{k} f_{2}^{k+1}\right)}{f_{1}+f_{2}} .
\end{gathered}
$$

Theorem 45. Let $m, n$ and $k$ be any integers. Then,

$$
\sum_{j=0}^{k} 2^{j} P_{n-2 m-m j, r}=2^{k+1} P_{n-(k+1) m, r}-P_{n, r}+m^{2} \alpha\left(2^{k+1}-1\right),
$$




$$
\begin{gathered}
\sum_{j=0}^{k}(-1)^{j} P_{n-m-2 m j, r}=\left\{\begin{array}{l}
\frac{1}{2} P_{n, r}+\frac{1}{2} P_{n-2 m(k+1), r}-\frac{1}{2} m^{2} \alpha, \quad \text { if } k \text { is even } \\
\frac{1}{2} P_{n, r}-\frac{1}{2} P_{n-2 m(k+1), r}, \quad \text { if } k \text { is odd }
\end{array}\right. \\
\sum_{j=0}^{k} 2^{k-j} P_{n+m-m j, r}=2^{k+1} P_{n, r}-P_{n-m(k+1), r}+m^{2} \alpha\left(2^{k+1}-1\right) .
\end{gathered}
$$

Proof. In the relation (identity (1.23) with the index $n$ shifted),

$$
P_{n, r}=2 P_{n-m, r}-P_{n-2 m, r}+\alpha m^{2},
$$

identify $f_{1}=2, f_{2}=-1, c=m, d=2 m$ and $\lambda=\alpha m^{2}$ and use these in Lemma 6 with $\left(X_{n}\right) \equiv\left(P_{n, r}\right)$.

\subsection{Binomial summation identities}

Lemma 7. Let $\left(X_{n}\right)$ be any arbitrary sequence. Let $X_{n}, n \in \mathbb{Z}$, satisfy a recurrence relation $X_{n}=f_{1} X_{n-c}+f_{2} X_{n-d}+\lambda$, where $f_{1}$ and $f_{2}$ are non-vanishing complex functions, not dependent on $n$, and $c$ and $d$ are integers. Then,

$$
\begin{gathered}
\sum_{j=0}^{k}\left(\begin{array}{l}
k \\
j
\end{array}\right) f_{1}^{j} f_{2}^{k-j} X_{n-k d+(d-c) j}=X_{n}-\frac{\lambda\left(\left(f_{1}+f_{2}\right)^{k}-1\right)}{f_{1}+f_{2}-1} \\
(-1)^{k} \sum_{j=0}^{k}(-1)^{j}\left(\begin{array}{l}
k \\
j
\end{array}\right) f_{2}^{k-j} X_{n+k(c-d)+d j}=f_{1}^{k} X_{n}+\frac{\lambda\left(\left(1-f_{2}\right)^{k}-f_{1}^{k}\right)}{1-f_{2}-f_{1}}
\end{gathered}
$$

and

$$
(-1)^{k} \sum_{j=0}^{k}(-1)^{j}\left(\begin{array}{l}
k \\
j
\end{array}\right) f_{1}^{k-j} X_{n+k(d-c)+c j}=f_{2}^{k} X_{n}+\frac{\lambda\left(\left(1-f_{1}\right)^{k}-f_{2}^{k}\right)}{1-f_{1}-f_{2}},
$$

for $k$ a non-negative integer.

Theorem 46. Let $m$ and $n$ be any integers and $k$ a non-negative integer. Then,

$$
\begin{gathered}
(-1)^{k} \sum_{j=0}^{k}(-1)^{j}\left(\begin{array}{l}
k \\
j
\end{array}\right) 2^{j} P_{n-2 m k+m j, r}=P_{n, r}-m^{2} k \alpha, \\
\sum_{j=0}^{k}\left(\begin{array}{l}
k \\
j
\end{array}\right) P_{n-m k+2 m j, r}=2^{k} P_{n, r}+2^{k-1} m^{2} k \alpha, \\
\sum_{j=0}^{k}(-1)^{j}\left(\begin{array}{c}
k \\
j
\end{array}\right) 2^{k-j} P_{n+m k+m j, r}=P_{n, r}-m^{2} k \alpha .
\end{gathered}
$$

Proof. Identify $f_{1}=2, f_{2}=-1, c=m, d=2 m$ and $\lambda=\alpha m^{2}$ (see proof of Theorem 45) and use these in Lemma 7 with $\left(X_{n}\right) \equiv\left(P_{n, r}\right)$. 
Theorem 47. Let $m$ and $n$ be any integers and $k$ a non-negative integer. Then,

$$
\begin{gathered}
(-1)^{k} \sum_{j=0}^{k}(-1)^{j}\left(\begin{array}{l}
k \\
j
\end{array}\right)(m+1)^{j} m^{k-j} P_{n-k(m+1)+j, r}=P_{n, r}-k \alpha T_{m} \\
\sum_{j=0}^{k}\left(\begin{array}{l}
k \\
j
\end{array}\right) m^{k-j} P_{n-k+(m+1) j, r}=(m+1)^{k} P_{n, r}+k(m+1)^{k-1} \alpha T_{m} \\
\sum_{j=0}^{k}(-1)^{j}\left(\begin{array}{c}
k \\
j
\end{array}\right)(m+1)^{k-j} P_{n+k+m j, r}=m^{k} P_{n, r}-k m^{k-1} \alpha T_{m}
\end{gathered}
$$

Proof. Write identity (1.25) as

$$
P_{n, r}=(m+1) P_{n-m, r}-m P_{n-m-1, r}+\alpha T_{m} .
$$

Identify $f_{1}=m+1, f_{2}=-m, c=m, d=n-m-1$ and $\lambda=\alpha T_{m}$ and use these in Lemma 7 with $\left(X_{n}\right) \equiv\left(P_{n, r}\right)$.

\section{Some triangular-numbers-specific properties}

We will conclude this study by presenting some results which are specific to triangular numbers.

Theorem 48. Let $n$ be an integer. Then,

$$
T_{n} T_{n-1}=T_{T_{n}-1}+T_{T_{n-1}-1} .
$$

Proof. Since $T_{n}-T_{n-1}=n$ and $T_{n}+T_{n-1}=n^{2}$, we have

$$
T_{n}+T_{n-1}=T_{n}^{2}+T_{n-1}^{2}-2 T_{n} T_{n-1} .
$$

Thus,

$$
2 T_{n} T_{n-1}=\left(T_{n}^{2}-T_{n}\right)+\left(T_{n-1}^{2}-T_{n-1}\right)
$$

and the result follows.

The implication of Theorem 48 is that the product of any two consecutive triangular numbers is the sum of two certain triangular numbers.

On account of the summation identity (1.44), the identity given in Theorem 48 invokes the next result.

Corollary 49. If $k$ is an integer, then,

$$
\sum_{j=0}^{k}(-1)^{j} T_{j} T_{j-1}=(-1)^{k} \frac{T_{T_{k+1} T_{k-1}}}{T_{k}} .
$$

Next we give a recursive identity involving any three consecutive triangular numbers. 
Theorem 50. If $n$ is an integer, $n \neq 0, n \neq-1$, then,

$$
\frac{T_{n+1} T_{n-1}}{T_{n}}=\frac{2}{T_{n}} T\left(\frac{2}{T_{n}} T\left(\frac{2}{T_{n}} T\left(\frac{2}{T_{n}}\left(\cdots \frac{2}{T_{n}} T\left(\frac{T_{n+1} T_{n-1}}{T_{n}}\right) \cdots\right)\right)\right)\right) .
$$

Here we have written $T(j) \equiv T_{j}$.

Proof. Setting $m=1$ in identity (1.35) gives

$$
T_{n+1} T_{n-1}=T_{n}^{2}-T_{n}=2 T_{T_{n}-1} .
$$

But,

$$
T_{n+1} T_{n-1}=T_{n}^{2}-T_{n} \Rightarrow \frac{T_{n+1} T_{n-1}}{T_{n}}=T_{n}-1 .
$$

Using (8.2) in (8.1), we have

$$
T_{n+1} T_{n-1}=2 T_{\frac{T_{n+1} T_{n-1}}{T_{n}}}
$$

Thus,

$$
\frac{T_{n+1} T_{n-1}}{T_{n}}=\frac{2}{T_{n}} T_{\frac{T_{n+1} T_{n-1}}{T_{n}}}
$$

from which we get again

$$
\begin{gathered}
\frac{T_{n+1} T_{n-1}}{T_{n}}=\frac{2}{T_{n}} T_{\frac{2}{T_{n}} T_{\frac{T_{n+1} T_{n-1}}{T_{n}}}}, \\
\frac{T_{n+1} T_{n-1}}{T_{n}}=\frac{2}{T_{n}} T_{\frac{2}{T_{n}} T_{\frac{2}{T_{n}}} T_{\frac{T_{n+1} T_{n-1}}{T_{n}}}}
\end{gathered}
$$

In short,

$$
\frac{T_{n+1} T_{n-1}}{T_{n}}=\frac{2}{T_{n}} T_{\frac{2}{T_{n}} T \frac{2}{T_{n}} \ldots}
$$

In view of identity (8.2), we have the continued fraction representation for $T_{n}$, given in the next theorem.

Theorem 51. If $n$ is an integer, $n \neq 0$, then,

$$
T_{n}=1+\frac{T_{n-1} T_{n+1}}{1+\frac{T_{n-1} T_{n+1}}{1+\ddots}}
$$

Theorem 52. If $k$ and $m$ are integers, $m \neq-1$, then,

$$
\sum_{j=1}^{k}(-1)^{j-1}\left(\frac{m-1}{m+1}\right)^{k-j} T_{j m}=(-1)^{k-1} T_{m} T_{k}
$$

Proof. Identity (1.32) is

$$
\frac{T_{j m}}{T_{m}}=T_{j}+\frac{T_{m-1}}{T_{m}} T_{j-1} .
$$

Multiply through by $\left(T_{m-1} / T_{m}\right)^{-j}$ to obtain

$$
\left(\frac{T_{m-1}}{T_{m}}\right)^{-j} \frac{T_{j m}}{T_{m}}=\left(\frac{T_{m-1}}{T_{m}}\right)^{-j} T_{j}+\left(\frac{T_{m-1}}{T_{m}}\right)^{1-j} T_{j-1} .
$$


In identity (1.45), choose

$$
f_{j}=\left(\frac{T_{m-1}}{T_{m}}\right)^{1-j} T_{j-1} .
$$

Theorem 53. If $k$ is a non-negative integer, then

$$
\sum_{j=1}^{k} \frac{1}{T_{j}}=\frac{T_{k-1}}{T_{k}}+1=\frac{2 k}{k+1}
$$

Proof. Set $n=j-1$ in identity (8.1) and rearrange

$$
T_{j-1}=T_{j-1}^{2}-T_{j-2} T_{j}
$$

Divide through by $T_{j} T_{j-1}$ to obtain

$$
\frac{1}{T_{j}}=\frac{T_{j-1}}{T_{j}}-\frac{T_{j-2}}{T_{j-1}}
$$

Now sum over $j$, making use of the telescoping summation formula (1.45) with $f_{j}=$ $T_{j-2} / T_{j-1}=(j-2) / j$.

Corollary 54. We have

$$
\sum_{j=1}^{\infty} \frac{1}{T_{j}}=2
$$

\section{References}

[1] K. Adegoke, Weighted sums of some second-order sequences, The Fibonacci Quarterly 56:3 (2018), 252-262.

[2] K. Adegoke, Weighted Tribonacci sums, Konuralp Journal of Mathematics 8:2 (2020), 355-360.

[3] C. K. Cook and M. R. Bacon, Some polygonal number summation formulas, The Fibonacci Quarterly 52:4 (2014), 336-343.

[4] E. Deza and M. M. Deza, Figurate Numbers, World Scientific, 2012.

[5] L. E. Dickson, History of the Theory of Numbers, Volume II: Diophantine Analysis, Dover Publications, 2005.

[6] A. S. Garge and S. A. Shirali, Triangular numbers, Resonance 17:7 (2012), 672-681.

[7] H. G. Gould, Combinatorial Identities: Table I: Intermediate Techniques for Summing Finite Series, http://www.math.wvu.edu/ gould/Vol.4.PDF.

[8] V. E. Hoggatt, JR., and M. Bicknell, Triangular numbers, The Fibonacci Quarterly 12:3 (1974), 221-230. 